\title{
Differentially Expressed Protein Analysis of Placenta from Women with Gestational Diabetes Mellitus using Tandem Mass Tag Quantitative Proteomics
}

\author{
Sun $X^{1 *}$, Qu T $T^{2}$, Yang $X^{1}, \operatorname{He} X^{1}, \mathbf{X u} X^{2}$, Mao $Y^{1}$, Guo \\ $\mathbf{N}^{1}$, Lu $F^{1}$, Yang $L^{1}$ and $Z$ hang $H^{1}$ \\ ${ }^{1}$ Department of Obstetrics, Gansu Provincial Hospital, \\ China \\ ${ }^{2}$ Department of Biotherapy Center, Gansu Provincial \\ Hospital, China \\ *Correspondling author: Xiaotong Sun, Department of \\ Obstetrics, Gansu Provincial Hospital, Lanzhou, Gansu, \\ China
}

Received: J anuary 13, 2021; Accepted: February 01, 2021; Published: February 08, 2021

\begin{abstract}
Gestational Diabetes Mellitus (GDM) is one of the diseases occurring in pregnancy. Although normal postpartum glycometabolism can be restored in most patients with GDM, they have a significantly increased risk of developing complications in the future. In recent years, many studies on the screening of differentially expressed proteins have been performed in patients with GDM by means of proteomics, but the pathogenesis of GDM in the placenta was still unclear. Thus, using the Tandem Mass Tag (TMT) quantitative technology, we aimed to identify candidate biomarkers that could predict GDM occurrence early and provide targets for future therapy. Placenta samples were obtained from pregnant women immediately after delivery. Quantitative proteomics was performed using TMT isobaric tags and liquid chromatography-tandem mass spectrometry. Bioinformatic analysis was performed to elucidate the biological processes that these differentially expressed proteins were involved in. Thirtyfive differentially expressed proteins were identified between patients with GDM and normal pregnant women. Therein, 7 and 28 proteins were upregulated and downregulated, respectively. Differentially expressed proteins were mainly enriched in African trypanosomiasis pathway, hematopoietic cell lineage, gap junction, glucagon signaling pathway, and retinol metabolism. Insulin resistance induced by the excessively activated glucagon signaling pathway in the placenta may be one of the reasons for GDM onset. Among the 35 differentially expressed proteins, excluding 12 unknown proteins or antibodies, 17 of the remaining 23 proteins converged to the same protein-protein interaction network, indicating that a highly linked protein interaction network in the placenta of patients with GDM affected the occurrence of disease.
\end{abstract}

Keywords: Gestational diabetes mellitus; Proteome; Placenta; Insulin resistance; Glucagon

\section{Introduction}

Gestational Diabetes Mellitus (GDM) is a pregnancy-specific disease, characterized by the new onset of any degree of glucose intolerance during pregnancy, affecting $18 \%$ of pregnant women worldwide [1,2]. Compared with pregnant women from other regions, Asian women have a higher prevalence of GDM [3]. GDM increases the risk of adverse maternal and neonatal outcomes, such as cesarean section, macrosomia, stillbirth, preeclampsia, preterm birth, jaundice, respiratory morbidity, neonatal hypoglycemia, and so on [4-8]. Although the disease disappears after delivery, it can have longterm effects on the mother and child, who are at risk of developing type 2 diabetes, cardiovascular disease, and certain cancer [9-12].

To prevent harming the mother and fetus, recent studies have focused on identifying candidate biomarkers to early predict GDM occurrence during pregnancy and postpartum complications. Among them, proteomic technology has been widely applied to screen differentially expressed proteins in different samples of the placenta, peripheral and umbilical venous plasma, exosome, urine, and omental adipose tissue from patients with GDM [13-21]. Energy production, complement system, immune response, inflammation, metabolism, and insulin resistance were reported to be associated with GDM $[14,16,19,21]$. Several proteins, such as apolipoprotein E, coagulation factor IX, fibrinogen alpha chain, and insulin-like growth factor-binding protein 5 in serum, or CD59 and IL1RA in urine, could be used as early diagnostic biomarkers to predict GDM occurrence $[15,16]$.

Nutrients, waste material, and energy are exchanged between the fetus and mother via the placenta. Most symptoms of women with GDM disappear or are alleviated after placenta delivery, suggesting that placental proteins or factors, including hormones, probably play important roles in the occurrence and development of this condition $[22,23]$. The syncytiotrophoblast is the area where the placenta contacts the endometrium. However, no studies using high-throughput proteomic technology to screen differentially expressed proteins between patients with GDM and normal pregnant women have been conducted. Therefore, this study aimed to identify candidate biomarkers, using the Tandem Mass Tag (TMT) quantitative technology, that could early predict GDM occurrence and provide targets for future therapy. 


\section{Methods}

\section{Patient recruitment and sample collection}

This study was approved by the Ethical Committee of the Gansu Provincial Hospital (No. 2018-044). All participants agreed to the sample collection and provided written informed consent. All research was performed in accordance with relevant regulations. The inclusion criteria were as follows: provided informed consent, aged between 18 and 35 years, singleton pregnancy, absence of diabetes mellitus, hypertension, kidney disease, and cardiovascular disease before pregnancy, and not taking any medication. All participants were screened for GDM according to a 75-g Oral Glucose Tolerance Test (OGTT) at 24-28 weeks of gestation. Women with GDM were diagnosed and selected as cases with any one of the following two items: fasting $\geq 5.1 \mathrm{mmol} / \mathrm{L}$ or 2 -h post-load glucose $\geq 8.5 \mathrm{mmol} / \mathrm{L}$. All pregnant women with GDM were managed with diet and without insulin intervention during the pregnancy. Nine normal pregnant women (control group) and nine women with GDM (GDM group) were recruited in this study. Placental samples were obtained after the participants' delivery at the Gansu Provincial Hospital. The placentas were separated from the placental samples within 30 minutes, frozen immediately in liquid nitrogen, and stored at $-80^{\circ} \mathrm{C}$.

\section{Protein extraction, liquid chromatography-tandem mass} spectrometry (LC-MS/MS) and bioinformatic analysis

This study is a continuation of our previous study, and the protein extraction, digestion, TMT labeling, LC-MS/MS analysis, protein identification, and bioinformatic analysis were described as before [24].

In brief, three placenta samples from normal pregnant women or the nine patients with GDM were randomly selected as one biological parallel. An equal amount of each sample was pooled, ground into powder, and homogenized in extraction buffer. Protein was extracted by sonication, filtered with $0.22-\mu \mathrm{m}$ filters, and quantified with the BCA Protein Assay Kit (Bio-Rad, USA). After protein digestion using the Filter-Aided Sample Preparation (FASP) procedure, three mixed samples from the control group were labeled with $126,127 \mathrm{~N}$, and $127 \mathrm{C}$ isobaric TMT tags, whereas the other three mixed samples from the patients with GDM were labeled with $128 \mathrm{~N}, 128 \mathrm{C}$, and $129 \mathrm{~N}$ isobaric TMT tags. The parameters of LC-MS/MS and data analyses, as well as the bioinformatic analysis of differentially expressed proteins, remain the same as before.

\section{Immunoblotting}

Placenta from the control group and GDM groups were homogenized in RIPA buffer with protease inhibitors and quantified using BCA Protein Assay Kit. Protein was separated in a 12\% SDS polyacrylamide gel and then transferred to a nitrocellulose membrane (Millipore, USA). After incubating at room temperature for $1 \mathrm{~h}$ in Tris-buffered saline containing $0.05 \%$ Tween-20 (TBST) with 5\% non-fat milk powder, the membrane was incubated at $4^{\circ} \mathrm{C}$ overnight in Anti-Polyadenylate-Binding Protein 4 (PABPC4, Proteintech, China), anti-Apolipoprotein A I (APOA1, Bioss, China), and AntiGlyceraldehyde-3-Phosphate Dehydrogenase (GAPDH, Proteintech) diluted in TBST with 5\% non-fat milk powder. After washing three times with TBST, the membrane was incubated at room temperature for $1 \mathrm{~h}$ with Horseradish Peroxidase (HRP)-conjugated secondary
Table 1: Clinical characteristics of the control and GDM groups.

Table 1: Clinical characteristics of the control and GDM groups.
\begin{tabular}{|l|c|c|c|}
\hline & Control & GDM & P value \\
\hline Sample size $(n)$ & 9 & 9 & \\
\hline Maternal age (years) & $30.33 \pm 4.15$ & $32.67 \pm 5.12$ & 0.274 \\
\hline Parity & $0.33 \pm 0.50$ & $0.44 \pm 0.53$ & 0.594 \\
\hline Pre-pregnancy BMI (kg/m²) & $22.11 \pm 2.02$ & $23.99 \pm 2.11$ & 0.14 \\
\hline Pregnancy BMI at 12 week (kg/m²) & $22.88 \pm 2.16$ & $25.76 \pm 1.56$ & $0.019^{*}$ \\
\hline Gestation age at delivery (week) & $39.67 \pm 1.07$ & $38.49 \pm 0.98$ & 0.068 \\
\hline Gestation age at 75-g OGTT (week) & $24.76 \pm 0.43$ & $24.70 \pm 040$ & 0.766 \\
\hline OGTT 0-h glucose (mmol/L) & $4.48 \pm 0.56$ & $5.46 \pm 0.64$ & $0.014^{*}$ \\
\hline OGTT 2-h glucose (mmol/L) & $6.61 \pm 0.92$ & $7.36 \pm 1.12$ & $0.028^{*}$ \\
\hline Insulin 0 h (mU/L) & $12.30 \pm 2.30$ & $11.73 \pm 1.79$ & 0.657 \\
\hline Insulin 2 h (mU/L) & $25.94 \pm 4.49$ & $92.15 \pm 15.83$ & $<0.001^{*}$ \\
\hline TC (mmol/L) & $4.91 \pm 0.98$ & $6.16 \pm 1.75$ & 0.172 \\
\hline TG (mmol/L) & $2.53 \pm 1.22$ & $3.56 \pm 0.95$ & 0.115 \\
\hline HDL (mmol/L) & $1.66 \pm 0.49$ & $1.79 \pm 0.11$ & 0.498 \\
\hline LDL (mmol/L) & $2.67 \pm 0.71$ & $2.97 \pm 0.36$ & 0.308 \\
\hline
\end{tabular}

antibody (1:5000, Biosharp, China). After washing another three times, protein bands were detected using SuperSignal West Pico Trial Kit (Thermo, USA) and imaged using Fusion FX (Vilber, France).

\section{Results}

\section{Clinical characteristics of the pregnant women}

The clinical characteristics of the control and GDM groups (Table 1). There were no significant differences in maternal age, parity, Body Mass Index (BMI) at pre-pregnancy, gestation age at delivery, gestation age at 75-g OGTT, and concentrations of fasting insulin, Total Cholesterol (TC), Triglyceride (TG), High Density Lipoprotein (HDL), and Low Density Lipoprotein (LDL) between the control and GDM groups. As expected, pregnancy BMI at 12 week and glucose levels at both time points of the OGTT were significantly higher in the GDM group $(\mathrm{p}<0.05)$. In detail, glucose levels at 0 - $\mathrm{h}$ and 2 - $\mathrm{h}$ OGTT in the GDM group were $5.46 \pm 0.64 \mathrm{mmol} / \mathrm{L}$ and $7.36 \pm 1.12 \mathrm{mmol} / \mathrm{L}$, respectively, whereas those in the control group were $4.48 \pm 0.56$ and $6.61 \pm 0.92 \mathrm{mmol} / \mathrm{L}$, respectively. The insulin concentration of $2-\mathrm{h}$ OGTT in the GDM group was $92.15 \pm 15.83 \mathrm{mU} / \mathrm{L}$, whereas that in the normal group was $25.94 \pm 4.49 \mathrm{mU} / \mathrm{L}$. GDM, gestational diabetes mellitus; BMI, body mass index; OGTT, oral glucose tolerance test; TC, total cholesterol; TG, triglyceride; HDL, high density lipoprotein; LDL, low density lipoprotein

\section{Identification and verification of differentially expressed proteins}

In this study, 35 differential expressed proteins were identified between the control and GDM groups (Table 2), according to fold change (average expression of GDM/control group) $\geq 1.2$ or $\leq 0.8$ and $\mathrm{p}<0.05$. Among them, 7 proteins were upregulated, and 28 proteins were downregulated. The K-means clustering of these differentially expressed proteins is shown in a heat map (Figure 1).

To confirm the results of the TMT analysis, immunoblotting was performed to verify the expression patterns of two proteins, PABPC4 and APOA1, in the same samples. GAPDH was chosen as an internal 
Table 2: The differentially expressed proteins identified in the placentas of patients with GDM and normal pregnant women.

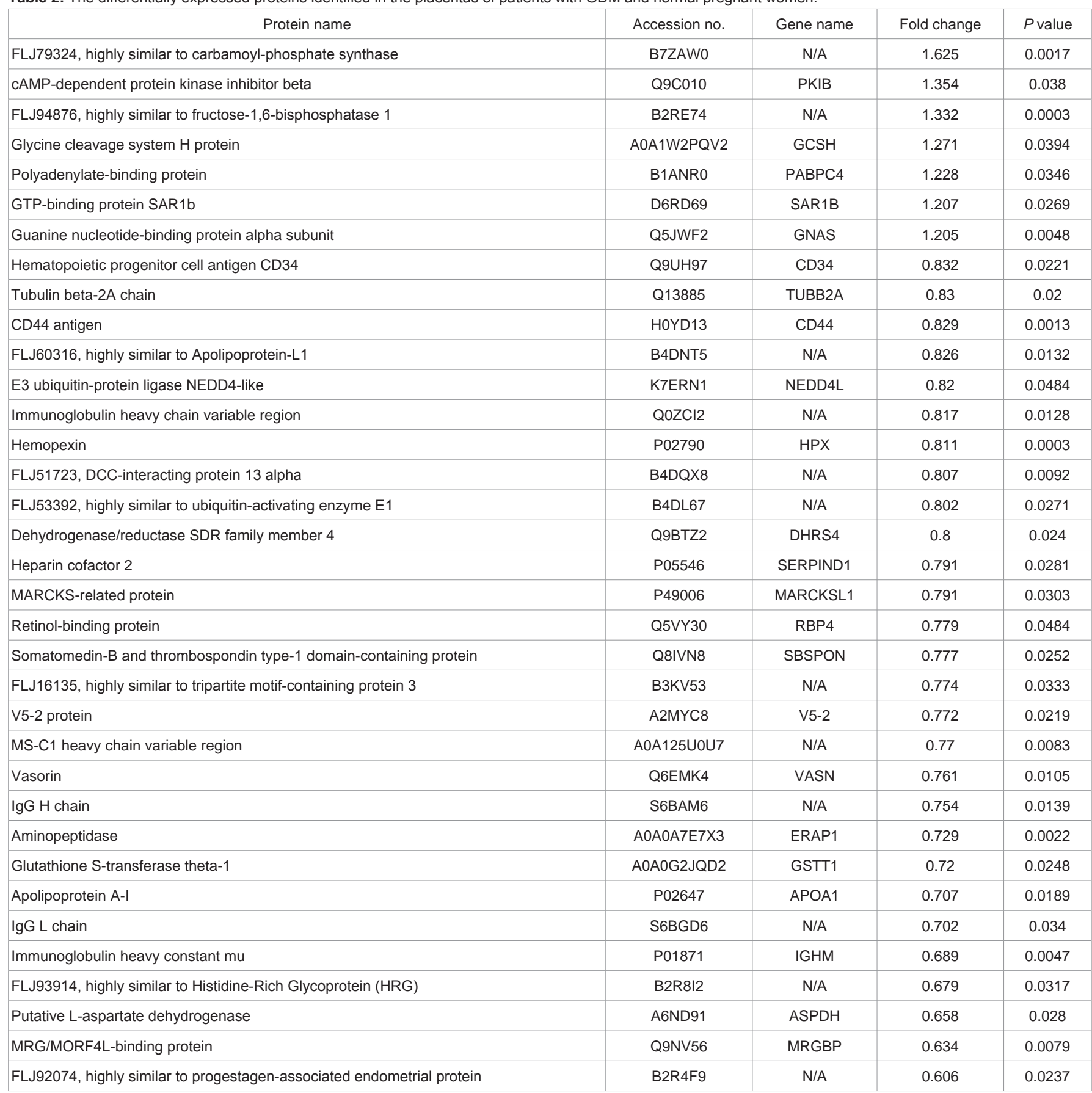

control. As shown in (Figure 2), in accordance with the proteomic results, the protein expression levels of PABPC4 were increased, and those of APOA1 were decreased in the GDM group than in the control group.

\section{Bioinformatic analysis}

The top 20 rankings of biological processes, molecular functions, and cellular components based on Gene Ontology (GO) annotation (Figure 3). The main biological process of these differentially expressed proteins was immune system process, endocytosis and import into cell, regulation of immune system process, positive regulation of immunoglobulin production, humoral immune response, complement activation, and immune response. The main molecular function of these proteins was beta- 2 adrenergic receptor binding, glycosaminoglycan binding, adrenergic receptor binding, quaternary ammonium group binding, phosphatidylcholine binding, and ammonium ion binding. The main cellular component was the extracellular region, part of the extracellular region, blood microparticle, extracellular space, extracellular exosome, and extracellular vesicle.

By Kyoto Encyclopedia of Genes and Genomes (KEGG) pathway 


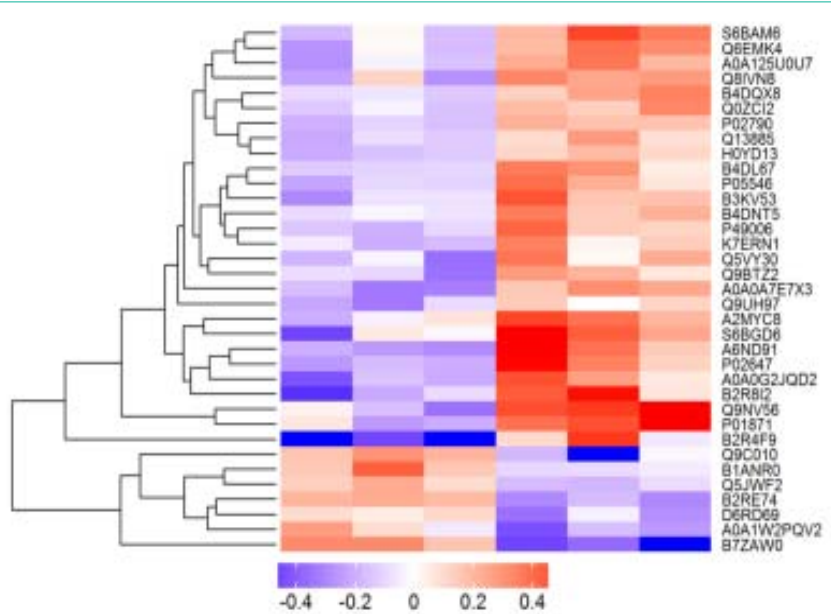

Figure 1: K-means clustering of differentially expressed proteins identified in human placenta.

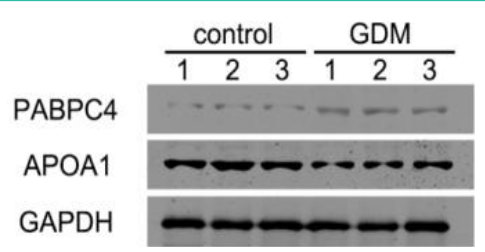

Figure 2: Immunoblotting analysis with anti-PABPC4, anti-APOA1, and antiGAPDH antibodies.

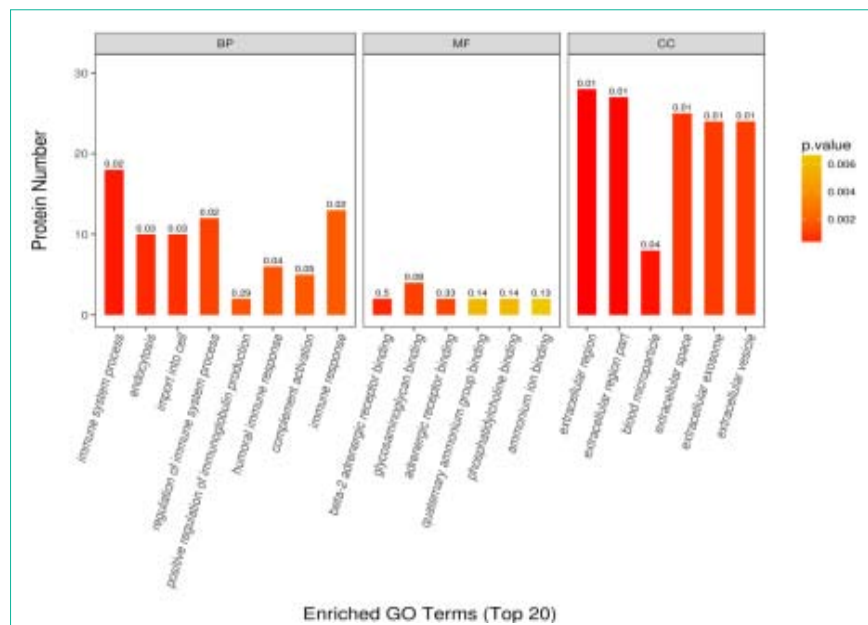

Figure 3: Bioinformatic analysis of the differentially expressed proteins. The top 20 rankings of biological process, molecular function, and cellular component that significantly changed based on GO analysis. The number on each bar represents the proportion of the differentially expressed proteins annotated to a GO category to the overall proteins annotated to the same GO category.

analysis, 9 differentially expressed proteins were enriched in African trypanosomiasis pathway, hematopoietic cell lineage, gap junction, glucagon signaling pathway, and retinol metabolism (Figure 4). By PPI analysis, among the 35 differentially expressed proteins, excluding 12 antibody proteins and unknown proteins, 17 of the remaining 23 differentially expressed proteins (PKIB, VASN, MRGBP, NEDD4L, PABPC4, ASPDH, SAR1B, TUBB2A, IGHM, GNAS, APOA1, SERPIND1, RBP4, HPX, MARCKSL1, CD44, DHRS4) interacted

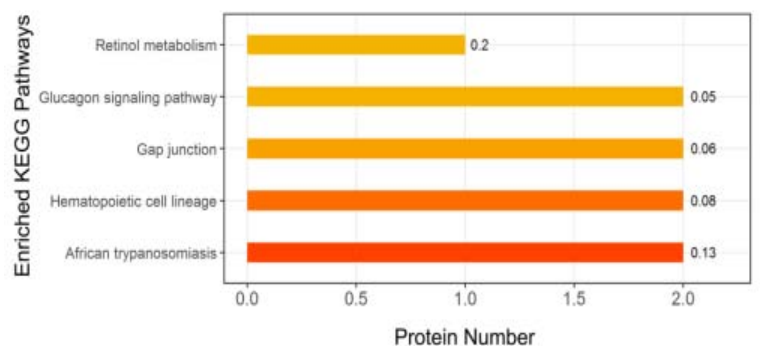

Figure 4: KEGG pathway enrichment of differentially expressed proteins. The number on each bar represents the proportion of differentially expressed proteins annotated to a KEGG pathway to the overall proteins annotated to the same KEGG pathway.

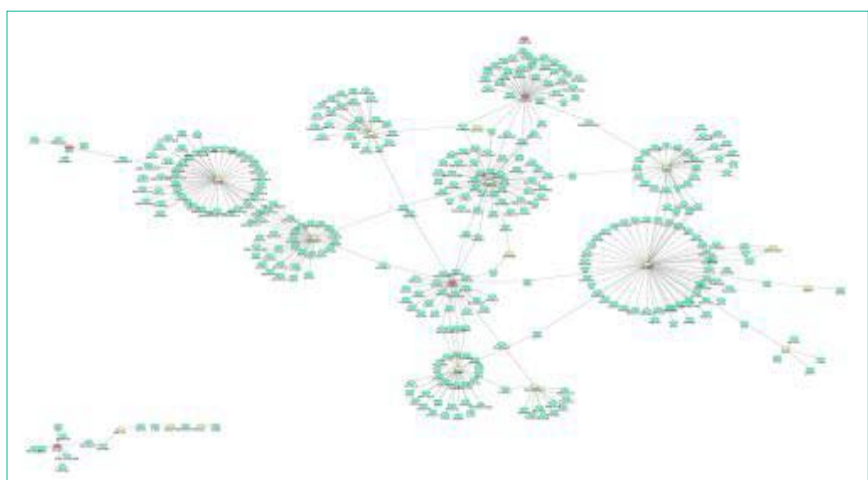

Figure 5: The protein interaction network of differentially expressed proteins. Red spot indicates upregulated protein, yellow spot indicates downregulated protein.

with each other and enriched into the same protein interaction network (Figure 5). These results suggested that a highly connected protein interaction network in the placenta of patients with GDM affects disease occurrence.

\section{Discussion}

Diabetes mellitus is a group of endocrine and metabolic diseases characterized by a high blood glucose level, and is classified into the following types: type 1 diabetes mellitus, Type 2 Diabetes Mellitus (T2D) and GDM, caused by a defect in insulin secretion or impairment in insulin function (insulin resistance). Similar to T2D, insulin resistance also exists in patients with GDM [25,26]; although different from T2D, the blood glucose level returns to normal immediately after childbirth, implying that the cause of insulin resistance in patients with GDM is in the placenta.

To clarify the pathogenesis and screen the potential biomarkers for GDM, we identified the differentially expressed proteins of women with GDM and compared these with those of normal pregnant women. The results showed that a total of 35 proteins were expressed differentially between the two groups. Of the 35 differentially expressed proteins, five have been reported to be implicated in diabetes mellitus. In our study, two of these proteins have an upregulated expression, whereas three proteins were downregulated.

cAMP-dependent Protein Kinase (PKA) is a pleiotropic enzyme that plays a role in several signaling pathways and cAMPmediated vasorelaxation through the cAMP second messenger. The mRNA and protein expression levels of PKA catalytic subunit were 
significantly decreased in streptozotocin-induced diabetic rats. The decreased expression levels lead to the decrease of PKA activity, which eventually leads to abnormal vasodilation in diabetic rats [27]. The protein expression level of PKIB was increased in our study, implying that it may cause some complications of GDM by inhibiting PKA activity. A recent study found that Adrenomedullin (ADM) dose-dependently inhibited synthesis and secretion of insulin, and circulating ADM concentrations were elevated in women with GDM. cAMP-dependent protein kinase inhibitor blocked the inhibitory effects of ADM [28]. Therefore, the increased expression of PKIB may be one of the reasons why insulin concentration in patients with GDM is higher than that in normal pregnant women.

Intrauterine hyperglycemia predisposed offspring of pregnant women with GDM to dyslipidemia, insulin resistance, and glucose intolerance through epigenetic modification-induced metabolic dysregulation. Whether the pancreas from the offspring of GDM mice model or umbilical cord blood from women with GDM, methylation analysis showed that the methylation levels of GNAS in the GDM group was significantly higher than that in normal pregnant women. These results suggested that maternal GDM-induced hypermethylation at GNAS and its effect on GNAS gene expression pattern may be associated with the increased risk of metabolic diseases in offspring $[29,30]$. In our study, the protein expression level of GNAS was increased in the placenta of women with GDM. In subsequent KEGG pathway analysis, this protein was enriched in glucagon signaling pathway. It is worthy of further study whether the expression of this protein is associated with the occurrence of metabolic diseases in later life of offspring.

Heparin Cofactor II (HCII), mainly produced in the liver and secreted into the bloodstream, is a novel vascular protective factor against atherosclerosis by inhibiting the activity of thrombin. HCII is capable of reversing insulin resistance and correlates inversely with fasting plasma glucose [31,32]. The decreased expression of HCII may increase the risk of cardiovascular complications in patients with insulin resistance syndrome. HCII, thus, could be a potential therapeutic target for T2D and GDM treatment.

RBP4 is an adipocyte-derived cytokine, which plays an important role in energy metabolism and insulin resistance. Many studies have shown that serum RBP4 concentration is increased in patients with GDM, suggesting that elevated serum RPB4 level is a risk factor for GDM pathogenesis [33-36]. However, our results showed that RBP4 protein expression in women with GDM was decreased. One reason is that serum RBP4 correlated with its mRNA expression in subcutaneous adipose tissue, rather than in visceral adipose tissue and placenta in patients with GDM [37] and RBP4 expression in the placenta may be regulated by negative feedback. APOA1 is the major protein component of HDL particles, which are associated with T2D development $[38,39]$. In this study, protein expression levels of APOA1 were found to be decreased in the placenta of women with GDM using TMT proteomic analyses and immunoblotting. We speculate that the low APOA1 level also plays a role in the potential pathogenesis of GDM.

Subsequently, bioinformatic analysis revealed that two upregulated proteins, GNAS and FLJ94876, were enriched in glucagon signaling pathway. As we have known, the homeostasis of blood glucose is regulated together by glucagon and insulin. Glucagon is responsible for elevating the plasma glucose concentration, whereas insulin does the opposite. Although clinical studies often focus on insulin dysfunction, glucagon plays an equally important role in T2D pathogenesis 40. Plasma glucagon levels were usually significantly higher in patients with GDM and T2D [40,41]. The upregulated expression of GNAS and FLJ94876 may lead to overactivation of glucagon signaling pathway, which may elevate the glucose concentration in placental and fetal blood flow. This also explains why GDM symptoms disappear after childbirth, because the over-activated glucagon signaling pathway disappears with placenta delivery. Further research and verification are needed to explore the role of glucagon signaling pathway in GDM pathogenesis.

Additionally, two downregulated proteins, APOA1 and FLJ60316, were enriched in African trypanosomiasis pathway. However, both are apolipoproteins, suggesting that abnormal lipid metabolism in the placenta is another risk factor for GDM.

Limitation of our study was that the sample size was small, for it is a little difficult to recruit more patients with strict inclusion criteria. A large number of experimental samples from different hospitals will be required to obtain further arguments in future study. In conclusion, thirty-five protein expressions were identified as significant differences between the control and GDM groups. Some of these differentially expressed proteins have been confirmed to be involved in the development of GDM. Our results show that insulin resistance in the placenta may be the potential mechanisms leading to the onset of GDM. Thus, these proteins can also be considered as candidate biomarkers for predicting or as an intervention target for preventing the onset of GDM.

\section{Acknowledgment}

This research was supported by Gansu Provincial Hospital (No. 19SYPYB-12 and 17GSSY2-3), Scientific Research Program of Health Industry in Gansu Province (No. GSWSKY2016-48), and Lanzhou Science and Technology Program (No. 2017-4-68). We thank all mothers for consenting to participate in this study and providing their information, Shanghai Applied Protein Technology Co. Ltd for providing technical support, and Editage for providing professional writing services.

\section{References}

1. Singh A, Subramani E, Datta Ray C, Rapole S, Chaudhury K. Proteomicdriven biomarker discovery in gestational diabetes mellitus: a review. J Proteomics. 2015; 127: 44-49.

2. Chiefari E, Arcidiacono B, Foti D, Brunetti A. Gestational diabetes mellitus: an updated overview. J Endocrinol Invest. 2017; 40: 899-909.

3. Yuen L, Wong VW. Gestational diabetes mellitus: Challenges for different ethnic groups. World journal of diabetes. 2015; 6: 1024-1032.

4. Farrar D, Duley L, Dowswell T, Lawlor DA. Different strategies for diagnosing gestational diabetes to improve maternal and infant health. The Cochrane database of systematic reviews. 2017; 8: CD007122.

5. Nerenberg KA, Johnson JA, Kaul P. PP157. Risk of preeclampsia in pregnant women with gestational diabetes in Alberta. Pregnancy Hypertens. 2012; 2: 323-324.

6. Gou BH, Guan HM, Bi YX, Ding BJ. Gestational diabetes: weight gain during pregnancy and its relationship to pregnancy outcomes. Chin Med $\mathrm{J}$ (Engl). 2019; 132: 154-160. 
7. Kumari R, Dalal V, Kachhawa G, Sahoo I, Khadgawat R, Mahey R, et al. Maternal and Perinatal Outcome in Gestational Diabetes Mellitus in a Tertiary Care Hospital in Delhi. Indian J Endocrinol Metab. 2018; 22: 116-120.

8. Hiersch L, Berger H, Okby R, Ray JG, Geary M, McDonald SD, et al. Gestational diabetes mellitus is associated with adverse outcomes in twin pregnancies. Am J Obstet Gynecol. 2019; 220: 102.e101-102.e108.

9. Leybovitz-Haleluya N, Wainstock T, Landau D, Sheiner E. Maternal gestationa diabetes mellitus and the risk of subsequent pediatric cardiovascular diseases of the offspring: a population-based cohort study with up to 18 years of follow up. Acta Diabetol. 2018.

10. Harreiter J, Dovjak G, Kautzky-Willer A. Gestational diabetes mellitus and cardiovascular risk after pregnancy. Women's health. 2014; 10: 91-108.

11. Fraser A, Lawlor DA. Long-term health outcomes in offspring born to women with diabetes in pregnancy. Curr Diab Rep. 2014; 14: 489.

12. Han KT, Cho GJ, Kim EH. Evaluation of the Association between Gestationa Diabetes Mellitus at First Pregnancy and Cancer within 10 Years Postpartum Using National Health Insurance Data in South Korea. International journal of environmental research and public health. 2018; 15: 2646.

13. Liao Y, Xu GF, Jiang Y, Zhu H, Sun LJ, Peng R, et al. Comparative proteomic analysis of maternal peripheral plasma and umbilical venous plasma from normal and gestational diabetes mellitus pregnancies. Medicine (Baltimore) 2018; 97: e12232.

14. Jayabalan N, Lai A, Nait S, Guanzon D, Scholz-Romero K, Palma C, et al. Quantitative Proteomics by SWATH-MS Suggest an Association Between Circulating Exosomes and Maternal Metabolic Changes in Gestational Diabetes Mellitus. Proteomics. 2019; 19: e1800164.

15. Guo Y, Han Z, Guo L, Liu Y, Li G, Li H, et al. Identification of urinary biomarkers for the prediction of gestational diabetes mellitus in early second trimester of young gravidae based on iTRAQ quantitative proteomics. Endocr J. 2018; 65: 727-735.

16. Zhao D, Shen L, Wei Y, Xie J, Chen S, Liang Y, et al. Identification of candidate biomarkers for the prediction of gestational diabetes mellitus in the early stages of pregnancy using ITRAQ quantitative proteomics. Proteomics Clin Appl. 2017; 11

17. Miao Z, Wang J, Wang F, Liu L, Ding H, Shi Z. Comparative proteomics of umbilical vein blood plasma from normal and gestational diabetes mellitus patients reveals differentially expressed proteins associated with childhood obesity. Proteomics Clin Appl. 2016; 10: 1122-1131.

18. Liu F, Zhao C, Liu L, Ding H, Huo R, Shi Z, et al. Peptidome profiling of umbilical cord plasma associated with gestational diabetes-induced fetal macrosomia. J Proteomics. 2016; 139: 38-44.

19. Ma Y, Gao J, Yin J, Gun L, Liu X, Chen S, et al. Identification of a Novel Function of Adipocyte Plasma Membrane-Associated Protein (APMAP) in Gestational Diabetes Mellitus by Proteomic Analysis of Omental Adipose Tissue. J Proteome Res. 2016; 15: 628-637.

20. Roverso M, Brioschi M, Banfi C, Visentin S, Burlina S, Seraglia R, et al. A preliminary study on human placental tissue impaired by gestational diabetes: a comparison of gel-based versus gel-free proteomics approaches. Eur J Mass Spectrom (Chichester). 2016; 22: 71-82.

21. Zhao C, Wang F, Wang $P$, Ding $H$, Huang $X$, Shi Z. Early second-trimeste plasma protein profiling using multiplexed isobaric tandem mass tag (TMT) labeling predicts gestational diabetes mellitus. Acta Diabetol. 2015; 52: 11031112.

22. Liao S, Vickers MH, Taylor RS, Fraser M, McCowan LME, Baker PN, et al. Maternal serum placental growth hormone, insulin-like growth factors and their binding proteins at 20 weeks' gestation in pregnancies complicated by gestational diabetes mellitus. Hormones. 2017; 16: 282-290.

23. Zhang B, Jin Z, Sun L, Zheng Y, Jiang J, Feng C, et al. Expression and correlation of sex hormone-binding globulin and insulin signal transduction and glucose transporter proteins in gestational diabetes mellitus placental tissue. Diabetes Res Clin Pract. 2016; 119: 106-117.

24. Sun X, Qu T, He X, Yang X, Guo N, Mao Y, et al. Screening of differentially expressed proteins from syncytiotrophoblast for severe early-onset preeclampsia in women with gestational diabetes mellitus using tandem mass tag quantitative proteomics. BMC Pregnancy Childbirth. 2018; 18: 437.

25. Zhu Y, Hedderson MM, Quesenberry CP, Feng J, Ferrara A. Central Obesity Increases the Risk of Gestational Diabetes Partially Through Increasing Insulin Resistance. Obesity (Silver Spring). 2019; 27: 152-160.

26. Montoro MN, Kjos SL, Chandler M, Peters RK, Xiang AH, Buchanan TA Insulin resistance and preeclampsia in gestational diabetes mellitus. Diabetes Care. 2005; 28: 1995-2000.

27. Matsumoto T, Wakabayashi K, Kobayashi T, Kamata K. Diabetes-related changes in cAMP-dependent protein kinase activity and decrease in relaxation response in rat mesenteric artery. Am J Physiol Heart Circ Physiol. 2004; 287: H1064-1071.

28. Dong Y, Banadakoppa M, Chauhan M, Balakrishnan M, Belfort M, Yallampalli C. Circulating Adrenomedullin Is Elevated in Gestational Diabetes and Its Role in Impaired Insulin Production by beta-Cells. J Clin Endocrinol Metab. 2019; 104: 697-706.

29. Zhu Z, Chen X, Xiao Y, Wen J, Chen J, Wang K, et al. Gestational diabetes mellitus alters DNA methylation profiles in pancreas of the offspring mice. Journal of diabetes and its complications. 2019; 33: 15-22.

30. Chen D, Zhang A, Fang M, Fang R, Ge J, Jiang Y, et al. Increased methylation at differentially methylated region of GNAS in infants born to gestational diabetes. BMC medical genetics. 2014; 15: 108.

31. Hasegawa Y, Ishigaki Y. Heparin Cofactor II: A Novel Plausible Link of Obesity and Diabetes with Thrombosis. Journal of atherosclerosis and thrombosis. 2017; 24: 1202-1203.

32. Kurahashi K, Inoue S, Yoshida S, Ikeda Y, Morimoto K, Uemoto R, et al The Role of Heparin Cofactor in the Regulation of Insulin Sensitivity and Maintenance of Glucose Homeostasis in Humans and Mice. Journal of atherosclerosis and thrombosis. 2017; 24: 1215-1230.

33. Du C, Kong F. A Prospective Study of Maternal Plasma Concentrations of Retinol-Binding Protein 4 and Risk of Gestational Diabetes Mellitus. Annals of nutrition \& metabolism. 2018; 74: 1-8.

34. Hu S, Liu Q, Huang X, Tan H. Serum level and polymorphisms of retinolbinding protein- 4 and risk for gestational diabetes mellitus: a meta-analysis. BMC Pregnancy Childbirth. 2016; 16: 52

35. Maghbooli Z, Hossein-Nezhad A, Mirzaei K, Karimi F, Besharati A, Omidfa $\mathrm{K}$, et al. Association between retinol-binding protein 4 concentrations and gestational diabetes mellitus and risk of developing metabolic syndrome after pregnancy. Reprod Sci. 2010; 17: 196-201.

36. Chan TF, Chen HS, Chen YC, Lee CH, Chou FH, Chen IJ, et al. Increased serum retinol-binding protein 4 concentrations in women with gestational diabetes mellitus. Reprod Sci. 2007; 14: 169-174

37. Kuzmicki M, Telejko B, Wawrusiewicz-Kurylonek N, Nikolajuk A, ZwierzGugala D, Jelski W, et al. Retinol-binding protein 4 in adipose and placental tissue of women with gestational diabetes. Gynecol Endocrinol. 2011; 27: 1065-1069.

38. Wu X, Yu Z, Su W, Isquith DA, Neradilek MB, Lu N, et al. Low levels of ApoA1 improve risk prediction of type 2 diabetes mellitus. J Clin Lipidol. 2017; 11: 362-368.

39. Riaz S, Alam SS, Akhtar MW. Proteomic identification of human serum biomarkers in diabetes mellitus type 2. J Pharm Biomed Anal. 2010; 51 1103-1107.

40. Haedersdal S, Lund A, Knop FK, Vilsboll T. The Role of Glucagon in the Pathophysiology and Treatment of Type 2 Diabetes. Mayo Clinic proceedings. 2018: 93: 217-239.

41. Grigorakis SI, Alevizaki M, Beis C, Anastasiou E, Alevizaki CC, Souvatzoglou A. Hormonal parameters in gestational diabetes mellitus during the third trimester: high glucagon levels. Gynecol Obstet Invest. 2000; 49: 106-109. 